\title{
Hyperchaotic Dynamics for Light Polarization in a Laser Diode
}

\author{
Cristian Bonatto \\ Instituto de Física, Universidade Federal do Rio Grande do Sul, 91501-970 Porto Alegre, Brazil
}

(Received 8 August 2017; revised manuscript received 23 October 2017; published 16 April 2018)

\begin{abstract}
It is shown that a highly randomlike behavior of light polarization states in the output of a free-running laser diode, covering the whole Poincaré sphere, arises as a result from a fully deterministic nonlinear process, which is characterized by a hyperchaotic dynamics of two polarization modes nonlinearly coupled with a semiconductor medium, inside the optical cavity. A number of statistical distributions were found to describe the deterministic data of the low-dimensional nonlinear flow, such as lognormal distribution for the light intensity, Gaussian distributions for the electric field components and electron densities, Rice and Rayleigh distributions, and Weibull and negative exponential distributions, for the modulus and intensity of the orthogonal linear components of the electric field, respectively. The presented results could be relevant for the generation of single units of compact light source devices to be used in low-dimensional optical hyperchaos-based applications.
\end{abstract}

DOI: 10.1103/PhysRevLett.120.163902

Since the development of the first laser [1,2] it has been known that the properties of the light emitted by laser devices are very different from that of conventional light, such as thermal light. While lasers have a high degree of coherence, conventional light has a high degree of incoherence. However, lasers may also exhibit irregular dynamics or erratic pulsation. For a long time, it was thought that noisy dynamics in laser devices were exclusively due to the effects of stochastic processes. From a fundamental point of view, this thinking was radically modified with the ideas introduced by Lorenz [3] with the discovery of the effects of sensitivity to the initial conditions in nonlinear systems, giving rise to the so-called deterministic chaos theory.

To describe the laser dynamics, theories were formulated taking into account the light-matter interaction inside an optical cavity, such as the semiclassical laser theory $[4,5]$. In 1975, Haken showed that the resulting set of equations from this theory, the Maxwell-Bloch equations, are fully equivalent to the atmospheric circulation model proposed by Lorenz, in which chaotic dynamics was discovered [6]. Haken's discovery paved the way to investigate chaotic behavior in optical systems. However, most of the lasers have very different relaxation rates, requiring an external perturbation to induce chaotic dynamics, as shown earlier by Arecchi et al. [7]. In the following years, nonlinear and chaotic dynamics in lasers became an important area of study $[8,9]$.

A very important class of lasers, especially for technological applications, are the semiconductor lasers (or laser diodes) [10-12]. Semiconductor lasers belong to class B [13], where the polarization dynamics can be discarded in a process called adiabatic elimination [14,15], and typically, they exhibit only damped oscillations in a free-running operation. To exhibit chaotic dynamics, these devices need some external perturbation, such as current modulation, optical injection or optical feedback, which has been extensively studied in the past, and it is still an ongoing research topic [16-18]. The dynamical behavior of semiconductor lasers changes considerably when the vector character of the electric field is taken into account and two macroscopic polarization modes compete inside the optical cavity. This is the case of a particular type of semiconductor laser, the vertical-cavity surface-emitting laser (VCSEL) [19]. Because of its specific geometry, the VCSEL can emit light with different polarizations. The additional polarization mode adds extra degrees of freedom in the solitary laser diode, enabling a very rich dynamics, including polarization switching and periodic and quasiperiodic oscillations. Recently, it was shown that deterministic polarization chaos in VCSELs [20] leads to a chaotic attractor that, when looked at the Poincaré sphere, exhibits a typical behavior of systems with 3 degrees of freedom, such as the so-called Lorenz chaos [3] or Lorenz-Haken chaos [6].

The aim of this Letter is to report the discovery of a new deterministic dynamical behavior in semiconductor lasers, i.e., a hyperchaotic phase, and analyze some statistical properties of the emitted light. It is shown that the hyperchaotic behavior can occur in a free-running operation, due to polarization mode competition, i.e., without any external perturbation. The deterministic light dynamics shown here is more intricate and irregular than the dynamics previously observed in free-running laser diodes, and it is beyond the Lorenz chaos, since it exhibits sensitivity to the initial conditions in two directions of phase space. Since the optical field consists of two orthogonal components in the plane transverse to the direction of light propagation, the effect of sensitivity to the initial conditions in two 
directions of the phase space can produce a highly disordered dynamics for the light polarization states. Another finding of this Letter is to show that the lowdimensional hyperchaotic flows that rule the macroscopic laser dynamics lead to a lognormal statistical distribution for the light intensity and a number of other well-known statistical distributions for the laser variables. This behavior, typical of stochastic systems, is shown here in a fully deterministic way.

The laser diode is modeled according to the widely used San-Miguel-Feng-Moloney model or spin-flip model given by the following set of rate equations $[21,22]$ :

$$
\begin{aligned}
\dot{E}_{x, y} & =\kappa(1+i \alpha)\left[(N-1) E_{x, y}+i n E_{y, x}\right]-\left(\gamma_{a}+i \gamma_{p}\right) E_{x, y}, \\
\dot{N} & =-\gamma\left[N(1+I)-\mu+i n\left(E_{y} E_{x}^{*}-E_{x} E_{y}^{*}\right)\right], \\
\dot{n} & =-\gamma_{s} n-\gamma\left[n I+i N\left(E_{y} E_{x}^{*}-E_{x} E_{y}^{*}\right)\right] .
\end{aligned}
$$

Here, $E_{x}$ and $E_{y}$ represent the slowly varying complex amplitudes of the two orthogonal linearly polarized fields. $I=\left|E_{x}\right|^{2}+\left|E_{y}\right|^{2}$ is the total output power (laser intensity). The normalized carrier variables $N$ and $n$ are defined by $N=\left(\bar{n}_{+}+\bar{n}_{-}\right) / 2$ and $n=\left(\bar{n}_{+}-\bar{n}_{-}\right) / 2$, where $\bar{n}_{+}$and $\bar{n}_{-}$ are the corresponding normalized densities of electrons with spin-down and spin-up, respectively. Amplitude anisotropy is modeled through linear dichroism $\gamma_{a}$ and phase anisotropy is modeled by the linear birefringence $\gamma_{p}$. Other parameters are defined as follows: $\alpha$ is the linewidth enhancement factor, $\kappa$ is the optical field decay rate, $\gamma$ is the decay rate of $N, \gamma_{s}$ is the spin-flip relaxation rate, and $\mu$ is the normalized injection current ( $\mu=1$ at threshold). $\mu$ and $\gamma_{s}$ are varied in the numerical simulations. The remaining parameters are fixed at $\alpha=3, \kappa=300 \mathrm{~ns}^{-1}, \gamma_{p}=30 \mathrm{~ns}^{-1}$, $\gamma_{a}=0.5 \mathrm{~ns}^{-1}$, and $\gamma=1 \mathrm{~ns}^{-1}$. Integrations were done using the standard fourth-order Runge-Kutta scheme with a fixed time-step equal to $h=0.0005$.

A phase diagram illustrating various instabilities occurring in the laser diode model [Eqs. (1)], as a function of $\mu$ and $\gamma_{s}$, is shown in Fig. 1. The diagram was done by computing the Lyapunov exponents (LEs), using the method of Ref. [23]. The magnitude of the largest LE $\left(\lambda_{1}\right)$ or, if $\lambda_{1} \approx 0$, the magnitude of the second largest LE $\left(\lambda_{2}\right)$ is displayed. Positive exponents are codified in yellowred tones and negative exponents in gray tones, with black (dark color) being associated with zero value. In this way, the locus of the zero value of $\lambda_{2}$ separating the gray regions indicates the location where bifurcations occur. The black color locus in the left side shows the occurrence of polarization switching and the black color locus in the diagonal shows a transition to an elliptically polarized state. Alternatively, these transitions could be computed through numerical continuation methods $[24,25]$. By checking the magnitude of $\lambda_{2}$, regions with two positive LEs were found (shown by the hatched area), characterizing a hyperchaotic dynamics [26].

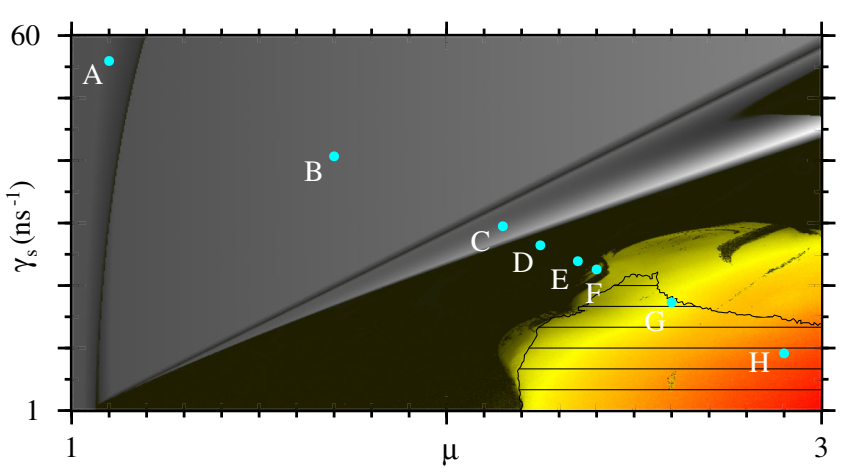

FIG. 1. Phase diagram of the laser diode as a function of normalized injection current, $\mu$, and decay rate of spin-flip processes, $\gamma_{s}$. Dynamical regimes for laser intensity: steady states (gray), periodic oscillations (dark), chaos (yellow-red), and hyperchaos (hatched area). See details in text. Parameter values for the marked points are $A\left(\mu, \gamma_{s}\right)=(1.10,56.0), B$ (1.70,41.0), $C(2.15,30.0), D(2.25,27.0), E(2.35,24.5), F$ (2.40,23.2), $G(2.60,18.0)$, and $H(2.90,10.0)$.

The main dynamical regimes for the light polarization states found in Fig. 1 are illustrated in Fig. 2. The polarization dynamics is shown in the Poincaré sphere, in terms of the normalized Stokes parameters (NSPs) $\bar{s}_{i}=$ $s_{i} / s_{0}[27,28]$. To better understand the overall dynamical scenario, especially the transitions shown in Figs. 2(d)-2(f),
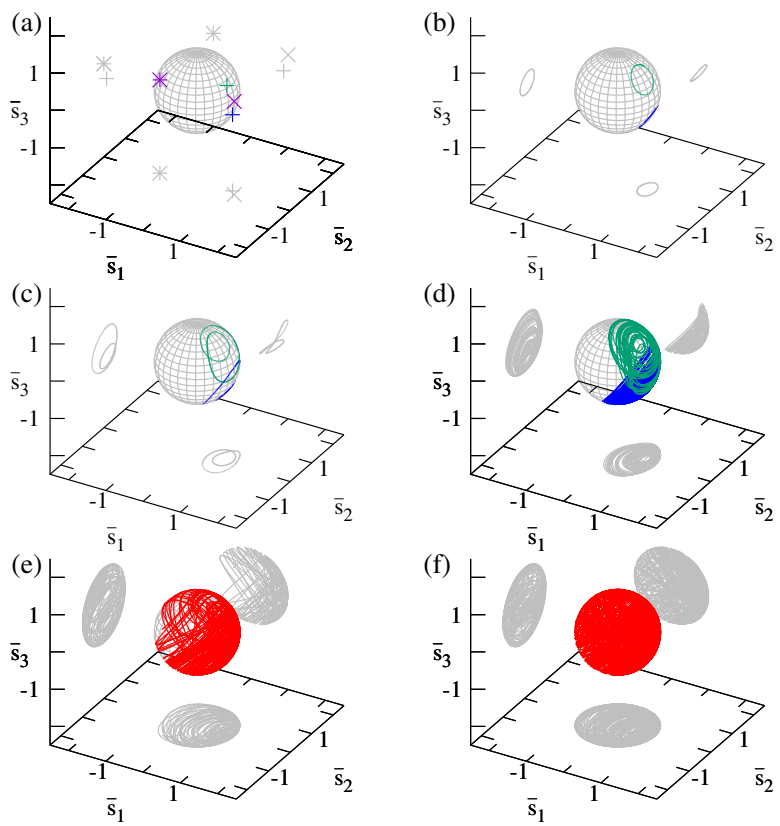

FIG. 2. Distinct dynamical regimes of light polarization states observed in the phase diagram shown in Fig. 1.(a) $(*)$ Linearly polarized light, $\hat{y} \mathrm{LP}$ mode, point $A,(\times)$ linearly polarized light $\hat{x}$ LP mode, point $B$, and (+) two elliptically polarized states, EP, point $C$; (b) two period-1 limit cycles, point $D$; (c) two period-2 limit cycles, point $E$; (d) two chaotic attractors, point $F$; (e) hyperchaotic dynamics, point $G$; (f) hyperchaotic dynamics, point $H$. The green attractor projections are not shown. 
it is important to analyze the behavior of the LEs along the instability processes. The system of Eqs. (1) is six dimensional and, therefore, has six associated LEs. The linearly polarized states, $\hat{x} \mathrm{LP}$ and $\hat{y} \mathrm{LP}$, and the elliptically polarized (EP) states, are stationary states for the intensity, but the field components oscillate periodically, where $\lambda_{1} \approx 0$ and the remaining LEs are less than zero. Note that LEs are estimated using finite-time integrations, so they are never exactly zero. When the EP state gets unstable, the intensity starts to oscillate periodically, but the fields oscillate in a quasiperiodic regime where $\lambda_{1,2} \approx 0$. When entering into the chaotic regime, one LE gets positive $\left(\lambda_{1}>0\right)$, but two LEs remain close to zero $\left(\lambda_{2,3} \approx 0\right)$. There are two EP states coexisting that destabilize giving rise to limit cycles that, in turn, give rise to two chaotic attractors, such as are illustrated in Figs. 2(a)-2(d). Eventually, the two chaotic attractors form a single large attractor, but, still, with only one positive LE. When going towards the hyperchaotic phase, along the chaos-hyperchaos transition, $\lambda_{2}$ becomes progressively positive, $\lambda_{3} \approx 0$, and $\lambda_{4}<0$. Going further into the welldeveloped hyperchaotic regime, $\lambda_{2}$ becomes significantly positive and $\lambda_{3,4} \approx 0$. These results are illustrated in Fig. 3 . Figure 3(a) displays the difference between the magnitude of $\lambda_{2}$ and $\lambda_{3}$. The hatched area in Fig. 1 marks the region where $\lambda_{2}>0.01$. Figures 3(b) and 3(c) show the four largest LEs by varying $\mu$ and $\gamma_{s}$, respectively. Figure 3(c) illustrates the evolution of $\lambda_{2}, \lambda_{3}$, and $\lambda_{4}$ along the continuous transition from chaos to hyperchaos, when decreasing $\gamma_{s}$. For certain fixed $\gamma_{s}$, the same continuous transition is observed along the chaos-hyperchaos or hyperchaos-chaos transition by increasing $\mu$. This is due to the fact that, when $\mu$ is increased, there is a transition from the hyperchaotic regime to a strong chaotic regime (i.e., $\lambda_{1}$ increases considerably, $\lambda_{2}$ approximates to zero, and $\lambda_{4}$ goes to negative values).

It is instructive to look at the probability density functions (PDFs) for the NSPs to better understand the disordered

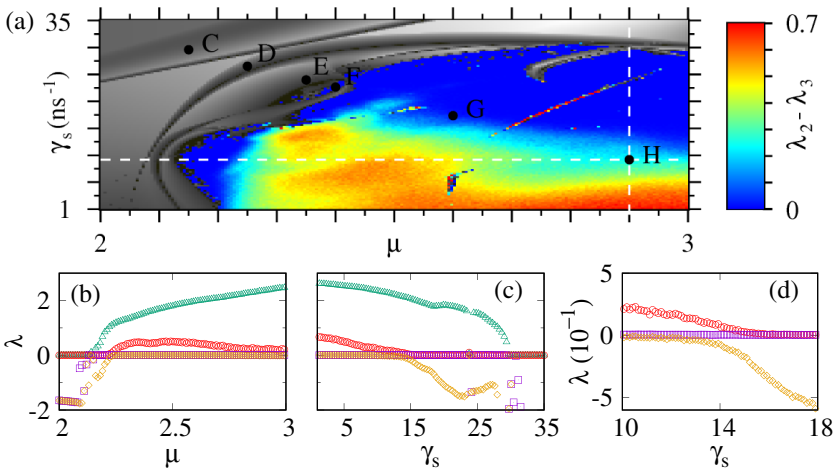

FIG. 3. (a) Magnification of the chaotic and hyperchaotic region in Fig. 1. The difference between $\lambda_{2}$ and $\lambda_{3}$ is shown in color scale. Gray shadings denote regular oscillations and display the magnitude of $\lambda_{3}$. (b) and (c) show the four largest LEs along the dashed lines in (a), where $\lambda_{1}$ is divided by five. (d) shows a magnification of (c) along the chaos-hyperchaos transition. states of hyperchaotic dynamics. For large $\gamma_{s}$, the polarization states over the Poincaré sphere are more concentrated around some specific positions and do not cover the whole sphere [Fig. 4(a)]. Also, for large $\mu$, the statistics of some NSPs shows pronounced peaks [Fig. 4(b)]. When going towards the hyperchaotic phase, this concentration starts to decrease, leading to more homogeneous distributions in certain parameter ranges [Figs. 4(c)-4(e)]. By increasing $\mu$, the distributions of the whole set of NSPs approximate to roughly flat distributions, where the variances are close to $1 / 3$, which is the variance of a uniform PDF from -1 to 1 [Figs. 4(f)-4(h)]. The degree of polarization decreases when increasing $\mu$, as can be checked by the computed fractional polarization (FP) [22] in Fig. 4. Alternatively, when going to the limit $\gamma_{s} \rightarrow \gamma$, considerably homogeneous distributions of the whole set of NSPs, and a decrease of the FP, can also be obtained. Notice that strong competition between polarization states and, therefore, strong reduction of the FP, have also been obtained in chaotic regimes of large $\gamma_{s}[22,24]$. However, for large $\gamma_{s}$ [as illustrated in Fig. 4(a)], the NSPs show distinct statistical properties when compared to the small $\gamma_{s}$ regime, even when $\mu$ is increased and the FP is reduced. This is because some of the NSPs cover only a portion of the Poincaré sphere or exhibit strongly bimodal statistical distributions. If truly rectangular statistical distributions were obtained for the whole set of NSPs, any instantaneous polarization state would have the same probability of occurring. The deterministic hyperchaotic polarized light, as shown here, can go very close to this limit.

Typical total output power for the hyperchaotic polarized light is shown in Fig. 5(a). Figure 5(b) shows the evolution of the hyperchaotic polarization states over the Poincare sphere (projected in a plane). The low-dimensional hyperchaotic flow covers, approximately, the whole surface of the sphere where the highly disordered light emission resembles an "unpolarized light" but in a fully deterministic
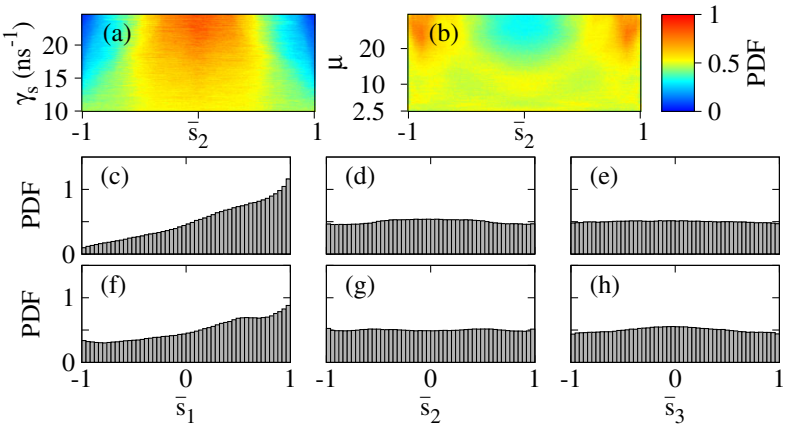

FIG. 4. PDFs of the normalized Stokes parameters. (a) $\mu=2.9$, (b) $\gamma_{s}=10$. From middle to bottom row, $\gamma_{s}=10$ and $\mu$ is equal to 2.9 and 4.2, corresponding to hyperchaotic regimes. The mean of each PDF is $\left(\left\langle\bar{s}_{1}\right\rangle,\left\langle\bar{s}_{2}\right\rangle,\left\langle\bar{s}_{3}\right\rangle\right)=(0.32,0.00,0.00)$ and $(0.19,0.00,0.00)$. The variance of each PDF is $\left(\sigma_{\bar{s}_{1}}^{2}, \sigma_{\bar{s}_{2}}^{2}, \sigma_{\bar{s}_{3}}^{2}\right)=$ $(0.26,0.32,0.33)$ and $(0.32,0.33,0.31)$. The fractional polarization is 0.10 , and 0.03 . 

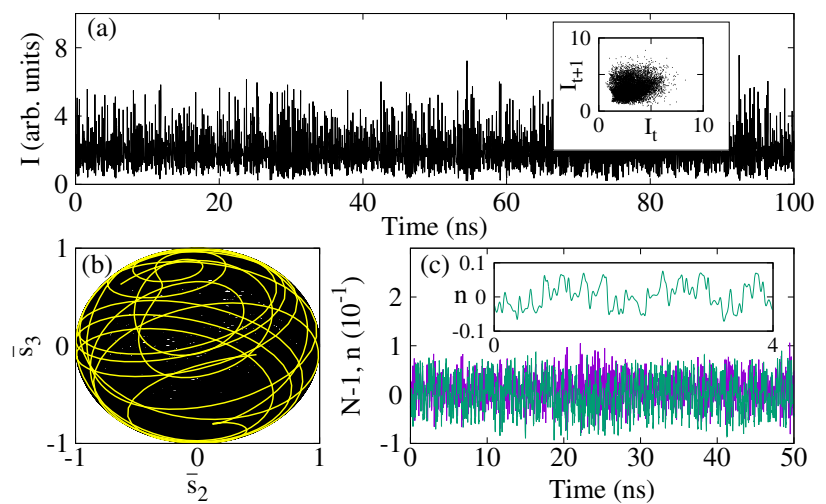

FIG. 5. (a) Total output power, $I$. The inset shows a return map of $I$ with 10000 pulse amplitudes. (b) In black: Spherical hyperchaotic attractor over the Poincaré sphere. In yellow: A trajectory of $1 \mathrm{~ns}$ in the hyperchaotic attractor. (c) Time traces of $N-1$ (purple) and $n$ (green). Parameters correspond to point $H$ in Fig. 1.

way. In fact, in a given instant of time, the light polarization is well defined, but the polarization states evolve in a very disordered way, spanning almost all possible states over the surface of the sphere, i.e., all orientation (azimuth) and ellipticity angles. The hyperchaotic dynamics arises for small $\gamma_{s}$, since the oscillations of $n$ start to be more important and, for certain levels of $\mu$, both modes become chaotic and interact in a complicated way. Since the modes are weakly coupled, the dynamics takes place in a global hyperchaotic attractor. In the hyperchaotic polarized light, the energy exchanges between the electric field and the two electron populations with different spins are equally important, leading the light polarization states to a highly disordered dynamics [Fig. 5(c)].

The statistical properties of the hyperchaotic light emission and other laser variables were analyzed and the main findings are shown in Fig. 6. Typically, it was found
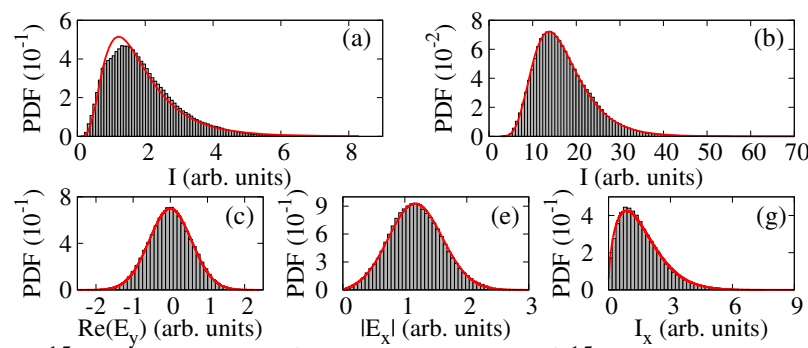
I (arb. units)
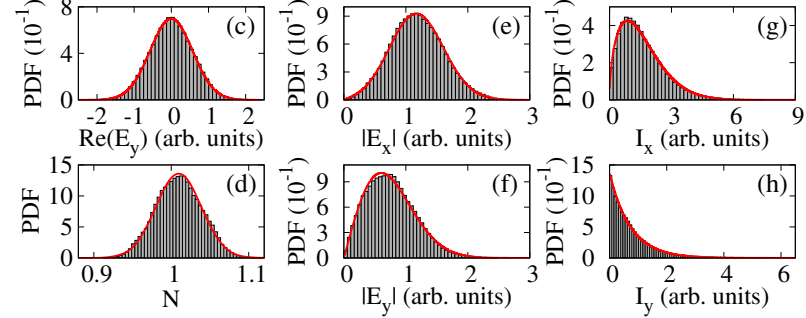

FIG. 6. PDFs of the laser variables. Parameters $\left(\mu, \gamma_{s}\right)$ are (a), (c), and (d) $(2.90,10)$, (b) $(18.00,15)$, (e) and (g) $(3.50,12)$, (f) and (h) $(3.05,10)$. Red curves show the respective fittings (see Supplemental Material [30] for details). that the PDFs for the total output power of the hyperchaotic light emission are well fitted by a lognormal PDF [Fig. 6(a)]. The agreement is not exact, but it is very close, as can be seen by the differences between the PDF of the laser intensity and the respective fitted curve (in red). When $\mu$ is increased, there is a transition from the hyperchaotic regime to a strong chaotic regime. In certain parameter ranges, it is possible to find very smooth distributions for the chaotic laser intensity, very well fitted by a lognormal PDF [Fig. 6(b)]. The lognormal statistics is a consequence of the very weak correlation between the two weakly coupled polarization modes. Note the difference between this statistics and that of VCSELs under optical feedback [29], where a high-dimensional dynamics, with some considerable degree of correlation, contribute to the light intensity dynamics. When looking at the dynamics of other laser variables, a number of known statistical distributions were found to describe the deterministic data from the laser system time series. Gaussian distributions for the electric field components and electron densities [Figs. 6(c) and 6(d)], Rice and Rayleigh distributions, and Weibull and negative exponential distributions for the modulus [Figs. 6(e) and 6(f)] and intensity [Figs. 6(g) and 6(h)] of the orthogonal linear components of the electric field, respectively, were found. All of them correspond to hyperchaotic regimes.

In conclusion, this work predicts that, besides polarization chaos found experimentally in Ref. [20], which shares properties of the Lorenz-Haken chaos, a free-running laser diode can also exhibit polarization hyperchaos with distinct statistical properties of light polarization dynamics in the Poincare sphere. The hyperchaotic polarized light is a consequence of the two-dimensional disorder of the two oscillatory modes in the field-matter coupling, explaining a highly randomlike behavior of polarization states, resembling a "randomly" polarized light, from a deterministic point of view. Usually, highly disordered light polarization states are associated with stochastically independent variables [31,32]. However, here, it was shown that the hyperchaotic dynamics produces a highly intricate and irregular laser emission with an output intensity exhibiting a deterministic lognormal statistical distribution and a number of other well-known statistical distributions for the deterministic dynamics of the laser variables. It is well known that a chaotic signal can have statistical properties which are consistent with random fluctuations. Some prominent examples are the " $U$ shape" PDF of the Ulam map and the uniform PDF of the tent map [33], and extreme events in chaotic systems [34], but, to the best of the author's knowledge, it is the first time that a variety of statistical distributions such as lognormal, Gaussian, Rice, Rayleigh, Weibull, and negative exponential-very common distributions in nature, usually associated with stochastic variables-has been shown being generated through a fully deterministic nonlinear process in a low-dimensional flow describing a 
physical system. The presented results are expected to have a large impact beyond the selected example (laser diode). It would be interesting to investigate correspondent analogies of the polarization instabilities described here in other vectorial flows, such as fluid dynamics and plasma physics. The results shown here could be of considerable relevance for chaos-based applications [35-39]. While low-dimensional chaotic systems are well-known to contain a series of regularities embedded in chaotic phases [40] leading to recurrent patterns with some degree of predictability [41], the hyperchaotic dynamics described here is more intricate and irregular than the conventional chaotic dynamics. By adding spontaneous emission effects for realistic values in VCSELs $[42,43]$, the hyperchaotic dynamics was found to be very robust [30].

C. B. thanks Alexandre Bonatto, Antonio Endler, and Sandra D. Prado for useful discussions.

[1] A. L. Schawlow and C. H. Townes, Phys. Rev. 112, 1940 (1958).

[2] T. H. Maiman, Nature (London) 187, 493 (1960).

[3] E. N. Lorenz, J. Atmos. Sci. 20, 130 (1963).

[4] W. E. Lamb, Jr., Phys. Rev. 134, A1429 (1964).

[5] H. Haken, Light (North-Holland, Amsterdan, 1985), Vol. 2.

[6] H. Haken, Phys. Lett. 53A, 77 (1975).

[7] F. T. Arecchi, R. Meucci, G. Puccioni, and J. R. Tredicce, Phys. Rev. Lett. 49, 1217 (1982).

[8] L. M. Narducci and N. B. Abraham, Laser Physics and Laser Instabilities (World Scientific, Singapore, 1988).

[9] P. Mandel, Theoretical Problems in Cavity Nonlinear Optics (Cambridge University Press, Cambridge, England, 1997).

[10] G. P. Agrawal and N. K. Dutta, Semiconductor Lasers (Kluwer Academic Publishers, Boston, 2001).

[11] W. Chow, S. Koch, and M. Sargent III, SemiconductorLaser Physics (Springer, Berlin, 1994).

[12] M. Yamada, Theory of Semiconductor Lasers (Springer, New York, 2014).

[13] J. R. Tredicce, F. T. Arecchi, G. L. Lippi, and G. P. Puccioni, J. Opt. Soc. Am. B 2, 173 (1985).

[14] L. A. Lugiato, P. Mandel, and L. M. Narducci, Phys. Rev. A 29, 1438 (1984).

[15] G.-L. Oppo and A. Politi, Europhys. Lett. 1, 549 (1986).

[16] D. M. Kane and K. A. Shore, Unlocking Dynamical Diversity: Optical Feedback Effects on Semiconductor Lasers (Wiley, New York, 2005).

[17] J. Ohtsubo, Semiconductor Lasers: Stability, Instability and Chaos (Springer, New York, 2005).

[18] Nonlinear Laser Dynamics: From Quantum Dots to Cryptography, edited by $\mathrm{K}$. Lüdge (Wiley-VCH, Weinheim, 2012).
[19] R. Michalzik, VCSELs: Fundamentals, Technology and Applications of Vertical-Cavity Surface-Emitting Lasers (Springer, New York, 2013).

[20] M. Virte, K. Panajotov, H. Thienpont, and M. Sciamanna, Nat. Photonics 7, 60 (2013).

[21] M. San Miguel, Q. Feng, and J. V. Moloney, Phys. Rev. A 52, 1728 (1995).

[22] J. Martin-Regalado, F. Prati, M. San Miguel, and N. B. Abraham, IEEE J. Quantum Electron. 33, 765 (1997).

[23] A. Wolf, J. B. Swift, H. L. Swinney, and J. A. Vastano, Physica (Amsterdam) 16D, 285 (1985).

[24] M. Virte, K. Panajotov, and M. Sciamanna, Phys. Rev. A 87, 013834 (2013).

[25] N. Li, H. Susanto, B. R. Cemlyn, I. D. Henning, and M. J. Adams, Phys. Rev. A 96, 013840 (2017).

[26] O. E. Rössler, Phys. Lett. 71A, 155 (1979).

[27] W. H. McMaster, Am. J. Phys. 22, 351 (1954).

[28] A. Molitor, S. Hartmann, and W. Elsäßer, Opt. Lett. 37, 4799 (2012).

[29] C-H Uy, D. Rontani, and M. Sciamanna, Opt. Lett. 42, 2177 (2017).

[30] See Supplemental Material at http://link.aps.org/ supplemental/10.1103/PhysRevLett.120.163902 for simulations with noise, hyperchaos for other VCSEL parameters, additional information and simulations about the statistical distributions, and hyperchaos characterization.

[31] E. Wolf, Introduction to the Theory of Coherence and Polarization of Light (Cambridge University Press, Cambridge, England, 2007).

[32] R. Barakat, J. Opt. Soc. Am. A 4, 1256 (1987).

[33] C. Beck and F. Schlögl, Thermodynamics of Chaotic Systems: An Introduction (Cambridge University Press, Cambridge, England, 1993).

[34] C. Bonatto, M. Feyereisen, S. Barland, M. Giudici, C. Masoller, José R. Rios Leite, and J. R. Tredicce, Phys. Rev. Lett. 107, 053901 (2011).

[35] G. D. VanWiggeren and R. Roy, Phys. Rev. Lett. 88, 097903 (2002).

[36] Handbook of Chaos Control, edited by E. Schöll and H. G. Schuster (Wiley-VHC, Weinheim, 2008).

[37] A. Uchida, Optical Communication with Chaotic Lasers: Applications of Nonlinear Dynamics and Synchronization (Wiley-VCH, Weinheim, 2012).

[38] L. Kocarev and S. Lian, Chaos-Based Cryptography: Theory, Algorithms and Applications (Springer, Berlin, 2011).

[39] M. Sciamanna and K. A. Shore, Nat. Photonics 9, 151 (2015).

[40] C. Bonatto, J. C. Garreau, and J. A. C. Gallas, Phys. Rev. Lett. 95, 143905 (2005).

[41] C. Bonatto and A. Endler, Phys. Rev. E 96, 012216 (2017).

[42] J. Mulet, C. R. Mirasso, and M. San Miguel, Phys. Rev. A 64, 023817 (2001).

[43] S. F. Yu, Analysis and Design of Vertical Cavity Surface Emitting Lasers (Wiley, Hoboken, 2003). 